\title{
A family with X-linked Cornelia de Lange syndrome due to a novel $S M C 1 A$ missense mutation identified by multi-gene panel sequencing
}

\author{
Sungwon Hong and Cha Gon Lee* \\ Department of Pediatrics, Nowon Eulji Medical Center, Eulji University, Seoul, Korea
}

\begin{abstract}
Cornelia de Lange syndrome (CdLS) is a rare, clinically and genetically heterogeneous, multi-system developmental disorder caused by mutations in genes that encode components of the cohesin complex. X-linked CdLS caused by an SMC1A mutation is an extremely rare disease characterized by phenotypes milder than those of classic CdLS. In the Republic of Korea, based on a literature review, one family with SMC1A-related CdLS with mild phenotypes has been genetically confirmed to date. In this study, we describe the clinical features of a Korean boy with a hemizygous novel missense mutation and his mother with a heterozygous mutation, i.e., c.2447G >A (p.Arg816His) in SMC1A, identified by multi-gene panel sequencing. The proband had a mild phenotype with typical facial features and his mother exhibited a mild, subclinical phenotype. This study expands the clinical spectrum of patients with X-linked CdLS caused by SMC1A variants. Moreover, these findings reinforce the notion that a dominant negative effect in a carrier female with a heterozygous mutation in SMC1A results in a phenotype milder than that in a male patient with the same mutation.
\end{abstract}

Key words: SMC1A, De Lange syndrome, X-Linked genes, High-throughput nucleotide sequencing.

\section{Introduction}

Cornelia de Lange syndrome (CdLS1-5; OMIM \# 122470, \#300590, \#610759, \#614701, \#300882)-a rare, clinically and genetically heterogeneous, multi-system developmental disorder-can be caused by mutations in 5 genes that encode regulators (NIPBL; OMIM \#608667, and HDAC8; OMIM \#300269) or subunits (SMC1A; OMIM \#300040, SMC3; OMIM \#606062, and RAD21; OMIM \#606462) of the cohesion complex [1-3]. Classic CdLS is characterized by distinctive facial features, including synophrys, long eyelashes, microcephaly, growth retardation, and upper-limb reduction defects. Although the exact incidence is unknown, CdLS likely affects 1 in 10,000 to 30,000 newborns.
The condition is probably underdiagnosed because affected individuals with mild or uncommon features may never be recognized as having CdLS. Mutations in NIPBL, SMC3, and RAD21 cause autosomal dominant CdLS, whereas mutations in SMC1A and $H D A C 8$ are associated with X-linked CdLS [2]. Approximately $5 \%$ of patients with CdLS have mutations in SMC1A $[1,2,4,5]$. In 2006, Musio et al. [6] first reported $4 \mathrm{CdLS}$-affected males with SMC1A mutations. To date, 56 different SMC1A variants have been reported, including 43 missense mutations, 7 small deletions, 2 splicing variants, 1 small insertion, 1 gross deletion, and 2 complex rearrangements, based on the Human Gene Mutation Database (HGMD Professional 2018.1, https://portal.biobaseinternational.com/hgmd/pro). SMC1A incompletely escapes X-

\footnotetext{
Received: May 4 2018, Revised: June 5 2018, Accepted: June 6 2018, Published: 30 June 2018

*Corresponding author: Cha Gon Lee, M.D. (iD http://orcid.org/0000-0001-7294-229X

Division of Child Neurology, Department of Pediatrics, Nowon Eulji Medical Center, Eulji University, 68 Hangeulbiseok-ro, Nowon-gu, Seoul 01830, Korea. Tel: +82-2-970-8222, Fax: +82-2-970-0068, E-mail: leechagon@eulji.ac.kr

Conflict of interest: The authors declare that they do not have any conflicts of interest.

(c) This is an open-access article distributed under the terms of the Creative Commons Attribution Non-Commercial License (http://creativecommons.org/licenses/by-nc/4.0/) which permits unrestricted non-commercial use, distribution, and reproduction in any medium, provided the original work is properly cited.

(c) Copyright 2018 by the Korean Society of Medical Genetics and Genomics

www.e-kjgm.org
} 
inactivation, and mutations in this gene have been identified in both hemizygous male and heterozygous female carriers [2,58]. In the Republic of Korea, based on a literature review, one family with SMC1A-related CdLS with milder phenotypes has been genetically confirmed to date [9]. We herein describe the clinical features of a Korean boy with a hemizygous novel missense mutation and his mother with a heterozygous mutation c. $2447 \mathrm{G}>\mathrm{A}$ (p.Arg816His) in SMC1A identified by multi-gene panel sequencing.

Written informed consent for the research investigation was obtained from the parents before participation. The Institutional Review Board of Eulji General Hospital in Seoul, Korea (IRB \#2014-06-007-001) approved the use of human clinical materials and blood in this study.

\section{Case}

\section{Patient}

A 33-month-old boy visited with recurrent seizures triggered by fever, which began around the age of 2 months. He was born prematurely at 36 weeks and 5 days via vaginal delivery, with a low birth weight of 2,490 g. His mother and father were nonconsanguineous individuals of Korean descent. He had a healthy younger brother. The patient suffered frequent infectious diseases, including recurrent viral pneumonia and acute pyelonephritis, beginning in the neonatal period. His parents reported that he exhibited mild psychomotor developmental delay since early infancy; he supported his head at 4 to 5 months, walked independently at 15 to 16 months, and used words with meaning at 13 to 14 months. On physical examination, his growth was within the normal range: weight, $13 \mathrm{~kg}(-1.01$ standard deviation [SD]); height, $89 \mathrm{~cm}(-1.56 \mathrm{SD})$; and head circumference, $49 \mathrm{~cm}$ (-0.62 SD). He had distinctive facial features, including synophrys, highly arched eyebrows, long, thick eyelashes, low-set ears, a wide nasal bridge, an upturned nasal tip with anteverted nares, and a microform cleft lip (Fig. 1). He had small hands but no limb deficiency. The patient exhibited apparent expressive language delays according to the serially checked Korean Developmental Screening Test for infants and children. Brain magnetic resonance imaging also revealed no remarkable findings. Electroencephalography showed a few multi-focal epileptiform discharges. The patient's hearing was normal, as were his echocardiogram and electrocardiography results. His serially checked abdominal ultrasound showed transient hydronephrosis of the left kidney.

\section{Patient's mother}

When the patient's mother visited with her son, she was 31 years old. She was the single child of healthy non-consanguineous Korean parents. She had no family history of neurologic diseases or developmental delay, except for her son. She had graduated high school and married at the age of 26 years. She had no notable intellectual disability, but her mother reported that she has a learning disability. Physical examination revealed that she had thicker eyebrows than her parents (Fig. 2), but there were no typical clinical features suggesting CdLS, with normal growth and the absence of microcephaly.

\section{Genetic analysis}

Genomic DNA was extracted from the patient's peripheral blood leukocytes and then sequenced on the Illumina MiSeq platform at Green Cross Genome (Yongin, Korea) using the TruSight One Sequencing Panel (Illumina, San Diego, CA, USA), which contains 4,813 Mendelian genes, including known genes associated with CdLS and several disorders with overlapping clinical features with CdLS, such as NIPBL, SMC1A, HDAC8, SMC3, RAD21, ARID1A, ARID1B, SMARCA4, SMARCB1, and $S M A R C E 1$. The minimal depth of coverage of the focused genes

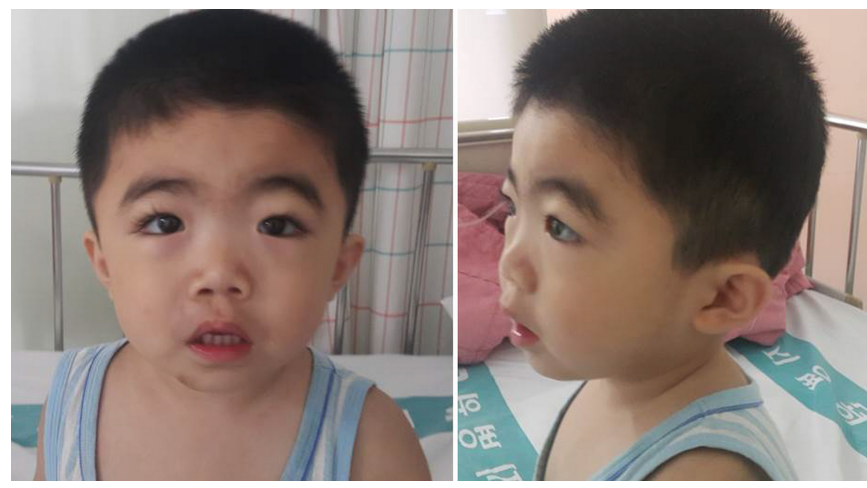

Fig. 1. Photographs of the patient. The patient's face showed a synophrys, highly arched eyebrows, long and thick eyelashes, wide nasal bridge, upturned nasal tip with anteverted nares, microform cleft lip, and low-set ears.

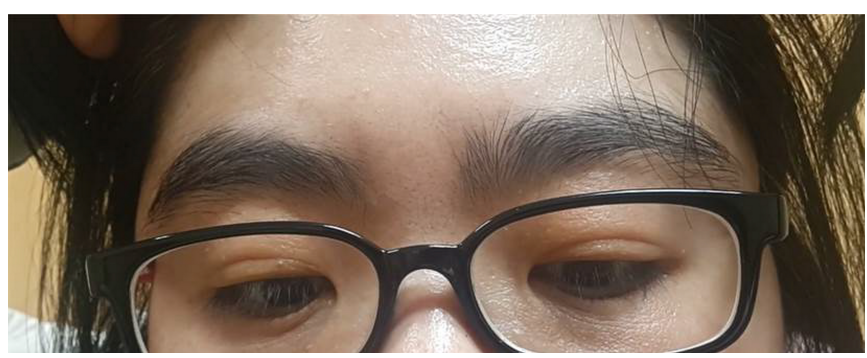

Fig. 2. Photograph of the patient's mother. Thick eyebrows were observed. 
was 73.9. The raw sequence reads were processed and aligned to the hg 19 human reference sequence.

The missense variant c. $2447 \mathrm{G}>\mathrm{A}$ (p.Arg816His) in SMC1A was identified. This variant has not been reported in the general population, including in the $1000 \mathrm{G}$ (http://browser.1000genomes. org/), gnomAD (http://gnomad.broadinstitute.org/), and ExAC (http://exac.broadinstitute.org/). Moreover, it has not been reported among groups of 622 and 1,100 individuals from the Korean Reference Genome Database (KRGDB, http://152.99.75.168/KRGDB/menuPages/introKor.jsp). This novel missense change occurred at an amino acid residue in which a missense change c.2446C $>\mathrm{G}$ (p.Arg816Gly) has been reported previously [10]. Thus, the novel missense variant was classified as a likely pathogenic variant according to guidelines of the American College of Medical Genetics and Genomics [11]. This variant was confirmed by Sanger sequencing of samples from the patient and his parents. The pedigree chart is presented in Fig. 3A, and the results of Sanger sequencing are presented in Fig. 3B. His mother possessed the heterozygous missense mutation c.2447G $>$ A (p.Arg816His).

\section{Discussion}

SMC1A encodes a protein belonging to a family involved in the structural maintenance of chromosomes. The SMC1 protein, as a structural component of the cohesin complex, is also thought to play important roles in stabilizing cellular genetic information, repairing damaged DNA, and regulating the activity of certain genes that are essential for normal development [4-7]. The X-linked SMC1A-related CdLS phenotype overlaps with, but is milder than, the phenotype associated with classic or severe CdLS; the phenotype retains many of the characteristic facial features but is not accompanied by predominant structural anomalies [4-6]. Our patient exhibited synophrys, long and thick eyelashes, microform cleft lip, small hands, fever-provoked seizures, a marked expressive language delay, and recurrent infections. With respect to the genotype-phenotype correlation for Korean patients with SMC1A-related CdLS, our patient and his mother consistently exhibited a mild phenotype with only the typical facial features and less severe growth, cognitive, and limb involvement [9].

Approximately 56 distinct SMC1A variants, mostly missense mutations, have been reported to date, and no mutation hotspots have been identified for CdLS. This novel missense mutation occurred at an amino acid residue where a different missense mutation classified as pathogenic has been previously

\section{A}

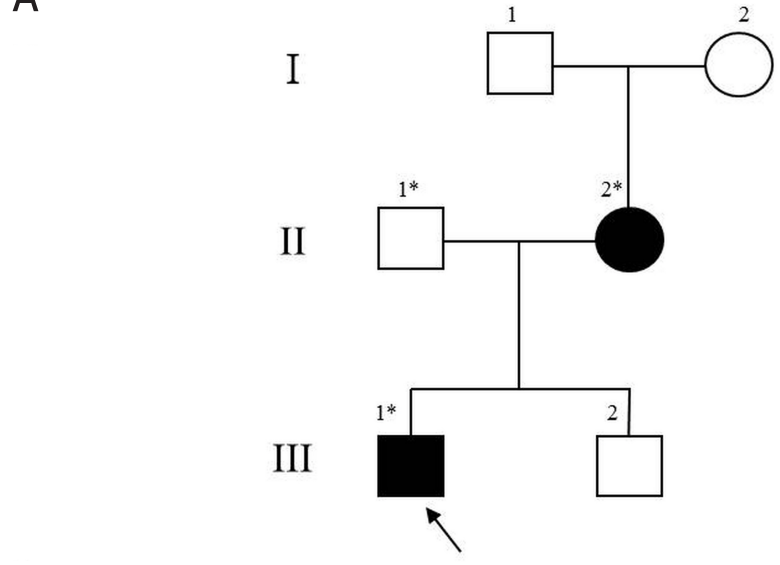

B

Father (II-1)

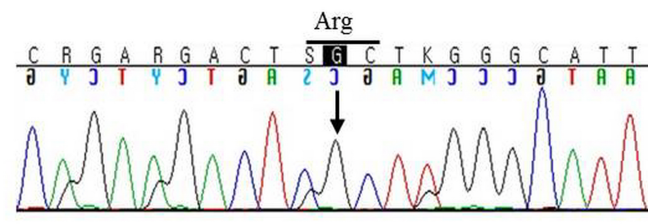

Mother (II-2)

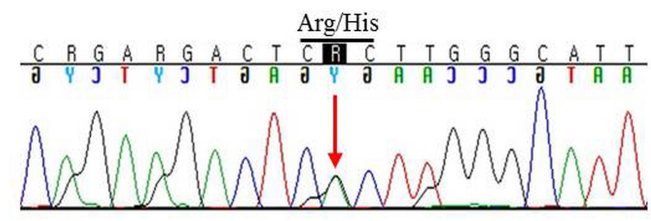

Patient (III-1)

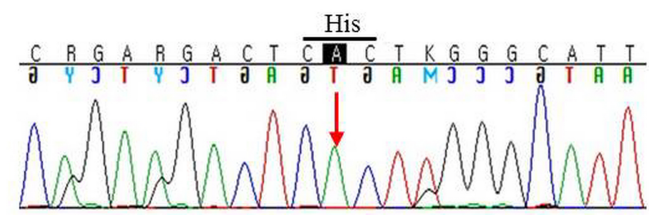

Fig. 3. Pedigree chart and results of Sanger sequencing. (A) Pedigree of the patient with Cornelia de Lange syndrome. The darkened symbol indicated by the black arrow represents the patient, "III-1." Symbols marked with central dots are definite carriers. Asterisks indicate sampled subjects. (B) Sanger sequencing of SMC1A using the genomic DNA of the patient and his parents.

reported [10]. The clinical characteristics of the present patient with a relatively mild phenotype reinforce the pathogenicity of the change in the amino acid residue at position 816.

SMC1A escapes $\mathrm{X}$-inactivation; mutations in this gene have been identified in both genders, with a notable predominance in females [10]. Thus, the disease presentation in affected females is attributed to the dominant-negative effect of the altered protein, rather than decreased protein levels or skewed X inactivation $[6,10]$. An unaffected or mild phenotype in a female carrier with the same SMC1A mutation as that in male patients has been previously reported [9]. These findings suggest that gender influences the correlation between genotype and phenotype 
in SMC1A-related CdLS [12]. The carrier mother in this study presented no typical clinical features suggesting CdLS and was clinically assessed only based on her affected son. The present results also reveal the disparate effects of the same mutation in carriers of different sexes, even among those belonging to the same family.

In summary, we described the clinical features of a Korean boy with a hemizygous novel missense mutation and his mother with a heterozygous mutation c. $2447 \mathrm{G}>\mathrm{A}$ (p.Arg816 His) in $S M C 1 A$. These results expand the clinical spectrum of patients with X-linked CdLS caused by SMC1A mutations. In particular, the findings highlight the distinct effects of the same variant in carriers of different sexes, even within the same family.

\section{Acknowledgements}

We would like to thank the patient's family for participating in the study.

\section{References}

1. Ansari M, Poke G, Ferry Q, Williamson K, Aldridge R, Meynert AM, et al. Genetic heterogeneity in Cornelia de Lange syndrome (CdLS) and CdLS-like phenotypes with observed and predicted levels of mosaicism. J Med Genet 2014;51:659-68.

2. Boyle MI, Jespersgaard $C$, Brøndum-Nielsen $K$, Bisgaard AM, Tümer Z. Cornelia de Lange syndrome. Clin Genet 2015;88:1-12.

3. Liu J, Krantz ID. Cornelia de Lange syndrome, cohesin, and beyond. Clin Genet 2009;76:303-14.

4. Borck G, Zarhrate M, Bonnefont JP, Munnich A, Cormier-Daire V, Col- leaux L. Incidence and clinical features of $\mathrm{X}$-linked Cornelia de Lange syndrome due to SMC1L1 mutations. Hum Mutat 2007;28:205-6.

5. Huisman S, Mulder PA, Redeker E, Bader I, Bisgaard AM, Brooks A, et al. Phenotypes and genotypes in individuals with SMC1A variants. Am J Med Genet A 2017;173:2108-25.

6. Musio A, Selicorni A, Focarelli ML, Gervasini C, Milani D, Russo S, et al. $\mathrm{X}$-linked Cornelia de Lange syndrome owing to SMC1L1 mutations. Nat Genet 2006;38:528-30.

7. Deardorff MA, Kaur M, Yaeger D, Rampuria A, Korolev S, Pie J, et al. Mutations in cohesin complex members SMC3 and SMC1A cause a mild variant of cornelia de Lange syndrome with predominant mental retardation. Am J Hum Genet 2007;80:485-94.

8. Mannini L, Cucco F, Quarantotti V, Krantz ID, Musio A. Mutation spectrum and genotype-phenotype correlation in Cornelia de Lange syndrome. Hum Mutat 2013;34:1589-96.

9. Jang MA, Lee CW, Kim JK, Ki CS. Novel pathogenic variant (c.3178G>A) in the SMC1A gene in a family with Cornelia de Lange syndrome identified by exome sequencing. Ann Lab Med 2015;35:639-42.

10. Liu J, Feldman R, Zhang Z, Deardorff MA, Haverfield EV, Kaur M, et al. SMC1A expression and mechanism of pathogenicity in probands with X-linked Cornelia de Lange syndrome. Hum Mutat 2009;30:1535-42.

11. Richards S, Aziz N, Bale S, Bick D, Das S, Gastier-Foster J, et al. Standards and guidelines for the interpretation of sequence variants: a joint consensus recommendation of the American College of Medical Genetics and Genomics and the Association for Molecular Pathology. Genet Med 2015;17:405-24.

12. Gervasini C, Russo S, Cereda A, Parenti I, Masciadri M, Azzollini J, et al. Cornelia de Lange individuals with new and recurrent SMC1A mutations enhance delineation of mutation repertoire and phenotypic spectrum. Am J Med Genet A 2013;161A:2909-19. 